\title{
Dipper, Christof, Schneider, Ute, Kartenwelten. Der Raum und seine Repräsentation in der Neuzeit
}

\section{Axelle Chassagnette}

\section{OpenEdition}

\section{Journals}

Édition électronique

URL : http://journals.openedition.org/ifha/1588

DOI : $10.4000 /$ ifha. 1588

ISSN : 2198-8943

Éditeur

IFRA - Institut franco-allemand (sciences historiques et sociales)

Référence électronique

Axelle Chassagnette, « Dipper, Christof, Schneider, Ute, Kartenwelten. Der Raum und seine

Repräsentation in der Neuzeit », Revue de l'IFHA [En ligne], Date de recension, mis en ligne le 01 janvier 2008, consulté le 22 septembre 2020. URL : http://journals.openedition.org/ifha/1588 ; DOI : https:// doi.org/10.4000/ifha. 1588

Ce document a été généré automatiquement le 22 septembre 2020.

(C)IFHA 


\title{
Dipper, Christof, Schneider, Ute, Kartenwelten. Der Raum und seine Repräsentation in der Neuzeit
}

\author{
Axelle Chassagnette
}

1 Un des topoï de l'histoire contemporaine de la cartographie, qui, sur ce point, s'appuie en particulier sur les travaux anglo-saxons de John Brian Harley ou de David Woodward, est de rappeler constamment le caractère construit des cartes, artefacts humains dont la fonction supposée - dans une perspective positiviste de l'histoire des sciences, aujourd'hui largement remise en cause - est de représenter la réalité, mais dont l'élaboration implique des omissions, un processus de sélection et de symbolisation des informations, et traduit les positions à la fois sociale, politique et éventuellement idéologique du cartographe à l'égard du sujet qu'il traite. C'est à cette tendance que se réfèrent C.D. et U.S., les éditeurs de l'ouvrage. Les articles publiés dans le recueil furent à l'origine des communications tenues à l'occasion du $45 \mathrm{e}$ Historikertag allemand de 2004 à Kiel, consacré au thème « Kommunikation und Raum » (« communication et espace »).

2 Les articles, qui couvrent une grande diversité thématique et chronologique, sont répartis en trois sections. La première, "Karten zeichnen und Karten lesen » (« dessiner et lire les cartes »), s'intéresse aux processus psychologiques que mettent en œuvre la lecture et la compréhension des cartes, ainsi qu'aux outils de représentation dont disposent les cartographes. Dans un premier article, U. Schneider traite de l'évolution des moyens graphiques mis en œuvre par la statistique européenne entre le début du XVIIIe et la fin du XIXe s., soulignant, notamment à la lumière de l'exemple des pratiques linguistiques - et dans une perspective proche des travaux menés en France par Morgane Labbé ou Gilles Palsky - la place croissante qu'y occupent les cartes. Dans une étude originale, V. Oswald étudie la production de deux atlas historiques de la firme Klett-Perthes au XXe s., et s'intéresse à la représentation bidimensionnelle de l'histoire, laquelle soulève des questions pratiques et théoriques. Sont ainsi analysés les problèmes rencontrés par la transposition en cartes d'un savoir 
discursif historique, problèmes qui relèvent de la sémiologie graphique, mais aussi de la hiérarchisation des connaissances. H.-D. Schultz expose les réflexions des pédagogues allemands du XIXe et du début du XXe s. sur l'usage des cartes dans l'enseignement de la géographie, soulignant que le recours à ce support a été très fréquemment discuté. Dans cette période qui est encore celle de la géographie " classique ", la relativité des discours et des positions portées par les cartes n'est jamais remise en cause : elles sont toujours comprises comme des représentations réalistes et objectives des paysages réels.

3 La seconde section porte le titre « Karten als Orientierungshilfe » (« Les cartes comme aide à l'orientation »). W. Behringer y détaille l'histoire de la carte des postes en Europe et plus particulièrement dans l'Empire à l'époque moderne (1500-1800), distinguée des cartes des routes et des itinéraires, de tradition antérieure, et des cartes des chemins de fer, et trace un parallèle entre cette histoire et celle du Grand Tour. D. Bitterling, dans un article dont les limites thématiques et chronologiques peuvent apparaître un peu floues, dresse un tableau de l'usage de la cartographie et de la statistique au service de l'administration et de l'économie dans l'état absolutiste de la France d'Ancien Régime, supposé porté par une vision uniformisatrice du territoire. L'étude est centrée sur la citation des discours et l'évocation succincte des grandes réalisations administratives de l'État. À l'exemple de la guerre des Boers de 1899-1902, A. Steinsieck met en lumière l'usage des cartes dans les médias publics anglais, montrant que la cartographie devient, au tournant du siècle, un support important de diffusion de l'information sur la guerre, et à ce titre un enjeu majeur pour le prestige et l'économie de la presse écrite.

Dans une dernière section intitulée « Karten als Text » (« Cartes comme textes »), dont l'homogénéité thématique est sans doute moins nette que dans les deux premières sections, G. Cecere s'interroge sur l'histoire de la frontière euro-asiatique, telle que la font apparaître les cartes russes et ouest-européennes au XVIIIe s. Étudiant le développement de la cartographie russe, l'auteur souligne que Pierre le Grand, en cherchant à conférer à la Russie une place sur la scène politique européenne, a voulu associer la Moscovie à ses proches voisins occidentaux, rejetant vers l'Asie les terres conquises à l'Est, en particulier la Sibérie : les cartographes et géographes russes, et à leur suite européens, ont fait de l'Oural la frontière naturelle entre Europe et Asie. Z. Laidlaw analyse le cheminement lent qui, au XIXe s., a conduit les cartographes britanniques à faire figurer l'Empire colonial en rouge sur les mappemondes, fréquemment utilisées comme outils d'administration coloniale, mais aussi diffusées par les médias de masse dans les dernières décennies du siècle. B. Struck étudie le tracé des frontières germano-polonaises sur les cartes allemandes produites entre 1800 et 1871 et montre qu'après 1848 - période de déploiement des idées nationales - ces documents tendirent à légitimer la domination sur la Pologne. Dans un dernier article, C. Dipper s'interroge sur la manière dont les cartes éditées après la Seconde Guerre mondiale retracent l'histoire du IIIe Reich et constate que, parallèlement à l'évolution de l'historiographie de cette période, les particularités de cette époque ne sont que lentement intégrées à la cartographie : figurant d'abord les attaques militaires et l'occupation menées par l'Allemagne, les bombardements dont furent victimes les Allemands, les atlas historiques de RFA n'ont fait apparaitre qu'à partir des années 1960 le thème de la solution finale - alors que les atlas édités en RDA continuaient à privilégier la représentation des mouvements de résistance au nazisme. 
5 Les articles de ce recueil, au-delà de leur diversité thématique et des sections dans lesquelles ils se trouvent cantonnés, ont en commun de traiter nombre de thèmes récemment développés dans l'histoire de la cartographie, comme les conditions de production matérielles, sociales et intellectuelles des documents, l'analyse de la lecture et de l'utilisation qui en sont faites, autant de thèmes qui ne font pas négliger la description et l'analyse fine des documents présentés. Si l'ouvrage peut apparaître comme une contribution supplémentaire à des problématiques déjà bien connues, il a le mérite de mettre à la disposition du lecteur une série d'études de qualité dont quelques-unes sont d'une grande originalité. On regrette l'absence d'une introduction plus étoffée et d'une conclusion qui auraient permis de conférer au recueil une plus grande unité problématique.

6 Axelle Chassagnette (MHFA) 